\title{
Enfermedad de Rosai Dorfman y Reactivación de Toxoplasmosis: Presentación de un Caso
}

\section{Rosai Dorfman's Disease and Reactivation of Toxoplasmosis: Case Report}

\author{
Gabriela Zambrano ${ }^{1}$, Felipe Mosquera ${ }^{2 *}$, Dra. María Cecilia Vivar ${ }^{3}$, Dr. Nicolás Vivar Diaz ${ }^{4}$ \\ 1 Facultad de Ciencias de la Salud Eugenio Espejo, Universidad Tecnológica Equinoccial, Quito, Ecuador. Médica \\ Internista Responsable de Control de Infecciones y Epidemiología del Hospital General Docente de Calderón. \\ 2 Facultad de Medicina, Pontificia Universidad Católica del Ecuador, Quito, Ecuador. Médico Tratante de la Unidad \\ de Medicina Interna del Hospital de Especialidades Carlos Andrade Marín. \\ ${ }^{3,4}$ Postgrado de Anatomía Patológica, Universidad Central del Ecuador, Quito, Ecuador \\ *fmosquera@puce.edu.ec \\ doi.org/ 10.26807/remcb.v40i1.654
}

Recibido 11-12-2017; Aceptado 23-03-2018

RESUMEN. - La enfermedad de Rosai Dorfman es una entidad clínica sumamente rara caracterizada por una proliferación histiocítica diseminada. Usualmente afecta a adultos jóvenes, presentándose con adenopatías múltiples por lo que constituye un diagnóstico diferencial de varias entidades infecciosas y linfoproliferativas. A continuación, presentamos un caso de esta enfermedad y una revisión sobre el tema.

Descripción del caso

Un paciente masculino de 32 años presentó adenomegalias generalizadas de forma recurrente acompañadas de fiebre, rash cutáneo episódico y toma del estado general. Al examen físico las adenopatías tienden a focalizarse en la región supraclavicular y axilar. Se realizó estudios de laboratorio los cuales no son relevantes salvo por niveles exagerados de IgG contra toxoplasma. En tomografía se encontró proliferación ganglionar diseminada a nivel cervical, torácico, axilar y abdominal. La biopsia de un ganglio supraclavicular derecho demostró la presencia de un infiltrado histiocítico con inmunohistoquimica positiva para S100, hallazgo compatible con la Enfermedad de Rosai Dorfman.

\section{Conclusiones}

La enfermedad de Rosai Dorfman es una entidad poco estudiada en cualquier medio que corresponde a un diagnóstico interesante en pacientes jóvenes con adenomegalias generalizadas, por lo que su presencia debe ser siempre considerada en casos atípicos o recurrentes como el expuesto en esta revisión.

PALABRAS CLAVES: Adenomegalias, enfermedades raras, histiocitosis, toxoplasmosis, Rosai-Dorfman

ABSTRACT. -Rosai Dorfman's disease is an extremely rare clinical entity characterized by a disseminated histiocytic proliferation. It usually affects young adults, presenting with multiple lymphadenopathy, which constitutes a differential diagnosis of several infectious and lymphoproliferative entities. Next, we present a case of this disease and a review on the subject.

Description of the case

A 32-year-old male patient presented recurrent generalized lymphadenopathy accompanied by fever, episodic skin rash and malaise. At examination he had several adenopathies located on the supraclavicular 
and axillary region. Laboratory studies were conducted which were not relevant except for exaggerated levels of IgG against toxoplasma. In tomography disseminated lymph node proliferation was found at the cervical, thoracic, axillary and abdominal levels. A biopsy of the right supraclavicular ganglion showed the presence of a histiocytic infiltrate with positive immunohistochemistry for S100, a finding compatible with Rosai Dorfman's disease.

\section{Conclusions}

Rosai Dorfman disease is an often ignored entity which constitutes an interesting differential diagnosis in young patients with generalized adenomegalies, so its presence should always be suspected in any atypical or recurrent cases such as the one presented in this review.

Keywords: Adenomegalies, histiocytosis, rare diseases, Rosai-Dorfman, Toxoplasmosis

\section{INTRODUCCIÓN}

La enfermedad de Rosai Dorfman o Histiocitosis Sinusal con Linfadenopatías Masivas (SHML por sus siglas en inglés) es una entidad clínica rara dentro de los trastornos de proliferación histiocítica. Las descripciones de SHML en la literatura son casi siempre realizadas en base de casos aislados o en raras ocasiones series de pocos pacientes. Esta enfermedad fue descrita inicialmente en el año de 1969 por el grupo de Rosai y Dorfman (Rosai y Dorfman 1969). Su epidemiología es poco clara con una incidencia global estimada de 690 casos desde su descripción. (NORD 2018). De acuerdo con la gran mayoría de observaciones la enfermedad suele presentarse en poblaciones pediátricas y en adultos jóvenes menores de 30 años con una predilección hacia los varones en un 58\% (Cai Yanan et al. 2017) (Piris et al. 2017), con una predilección por la etnia africana (Gomes Pinto et al 2008), aunque existen excepciones anecdóticas donde la enfermedad es diagnosticada en edades más tardías, reportándose casos aislados entre la sexta y séptima década de vida (Liao y Chiang 2010). El cuadro se considera benigno, sin embargo, existen casos en los cuales se ha reportado complicación usualmente por compresión de estructuras vecinas que ha requerido tratamiento quirúrgico. Adicionalmente la enfermedad puede infiltrar algunos tejidos sólidos con manifestaciones variables dependiendo del órgano afectado. Dada la naturaleza poco frecuente de la enfermedad, esta entidad debe ser considerada en pacientes jóvenes con adenomegalias sistémicas en medios donde coexisten otras infecciones de similares características como lo son la tuberculosis y el VIH. Es el objetivo del presente artículo ilustrar el caso de un paciente diagnosticado con Enfermedad de Rosai Dorfman asociada a reactivaciones recurrentes de toxoplasmosis en un adulto joven inmunocompetentente.

\section{DESCRIPCIÓN DEL CASO}

Se trata de un paciente masculino de 32 años de edad en quien de entre sus antecedentes personales se destaca la presencia de reactivaciones serológicas de toxoplasmosis, alergia a las sulfas y un antecedente quirúrgico de septoplastía de reducción nasal quien acude a la consulta por presentar adenomegalias de aparición intermitente, además de episodios de rash cutáneo de predominio en espalda desde hace cinco años. Describe que estos episodios se presentan de forma imprevista, aunque al parecer el estrés emocional puede precipitarlos. Usualmente las adenomegalias son localizadas a nivel de regiones cervicales y axilares y se acompañan de astenia, polimialgias y alza térmica cuantificada en más de $38^{\circ} \mathrm{C}$ por varias ocasiones y que cede espontáneamente. Niega la presencia de otros acompañantes junto a dichos episodios. Al examen físico impresiona la presencia de una adenomegalia supraclavicular indurada, no dolorosa a la palpación, adherente a planos profundos y de contextura renitente de aproximadamente $2 \mathrm{~cm}$ de diámetro.

En los exámenes de laboratorio iniciales se solicita una biometría hemática la cual no reporta hallazgos de relevancia, PCR y velocidad de sedimentación dentro de parámetros normales. La Prueba de Mantoux fue categorizada como anérgica. Estudios de VIH negativos y LDH en rangos normales, así como un despistaje de autoinmunidad realizado por medio de anticuerpos antinucleares y anticuerpos anti citoplasma de neutrófilos (ambos resultados se encuentran dentro de parámetros normales). Destaca dentro de la serología TORCH la cual reporta un incremento desmesurado de $\mathrm{IgG}$ contra Toxoplasma (>200000). Adicionalmente se realizó electroforesis de proteínas donde se evidenció hipergamaglobulinemia. 
Se solicita una tomografía de cuello y tórax donde se observan múltiples adenomegalias en los niveles cervicales II, III y IV bilaterales. Se describe también la presencia de pequeños ganglios en los niveles V. Se demuestran múltiples conglomerados ganglionares en los niveles torácicos 2, 3, 4, 5, 6, $7,8,9,10$. Estas adenomegalias difusas también se encuentran en la región para aórtica y axilar.

Inicialmente el paciente no tiene ninguna conducta adicional en relación a este cuadro considerándolo como reactivo, sin embargo, persiste con episodios intermitentes de alza térmica y adenomegalias difusas exacerbadas por episodios virales de vías respiratorias altas. Nuevamente se realizan estudios complementarios que arrojan resultados idénticos. Se plantea incluso en este caso en un determinado momento el inicio de tratamiento específico contra toxoplasmosis, sin embargo, por el historial de alergia sulfas se decide diferir el inicio de la medicación, tras lo cual la sintomatología nuevamente desaparece. Al presentarse nuevamente episodio semejante luego de tres meses, se reevalúa el caso y se decide obtener una pieza de biopsia para tener una mejor aproximación diagnóstica. Se procede a realizar una biopsia con exéresis ganglionar, acto que se realiza sin complicaciones, donde se identifica la presencia de una única adenomegalia supraclavicular derecha indurada. El estudio histopatológico reveló tres fragmentos de ganglio linfático con arquitectura parcialmente conservada, con ocasionales folículos linfoides de tamaño variable, los senos linfoides se encuentran dilatados (hiperplasia sinusal) y llenos de histiocitos,

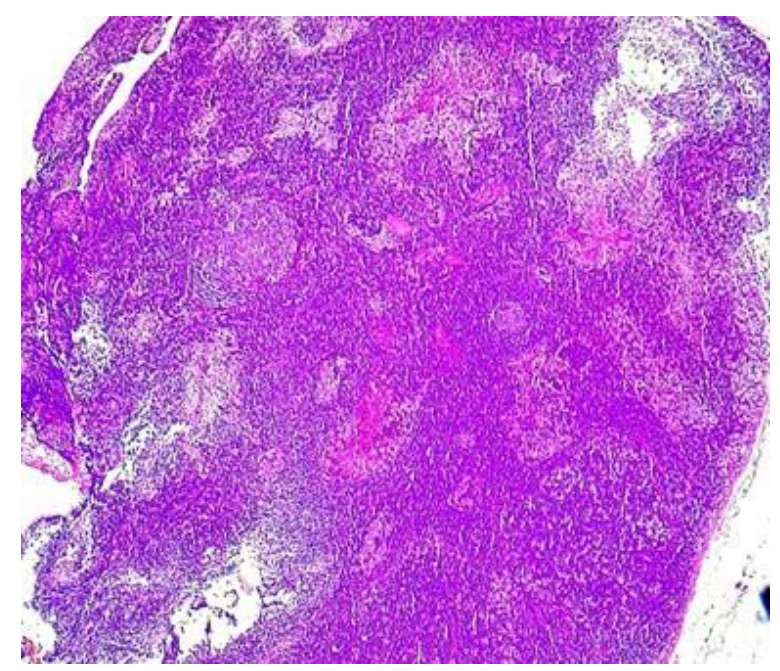

Figura 1: Corte histológico de ganglio linfático con pérdida parcial de la arquitectura. Sinusoides dilatados.

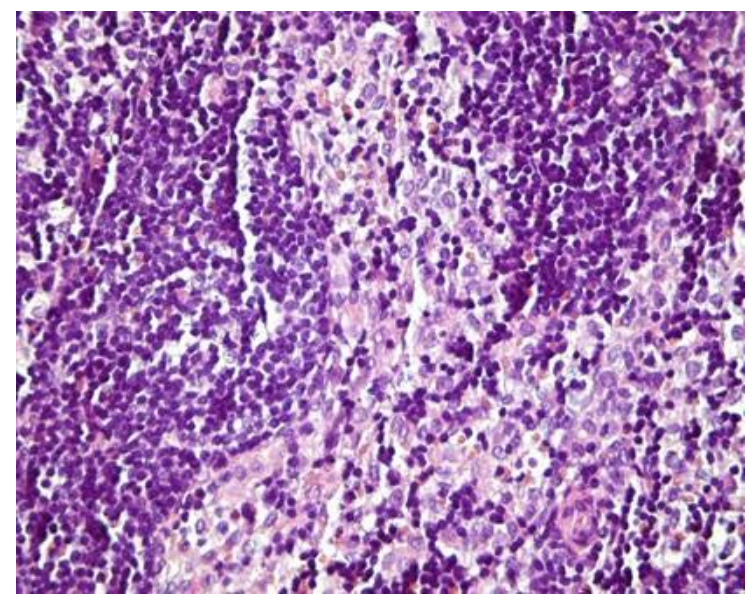

Figura 2: Corte histológico de ganglio linfático, sinusoides llenos de histiocitos de citoplasma vacuolado. 


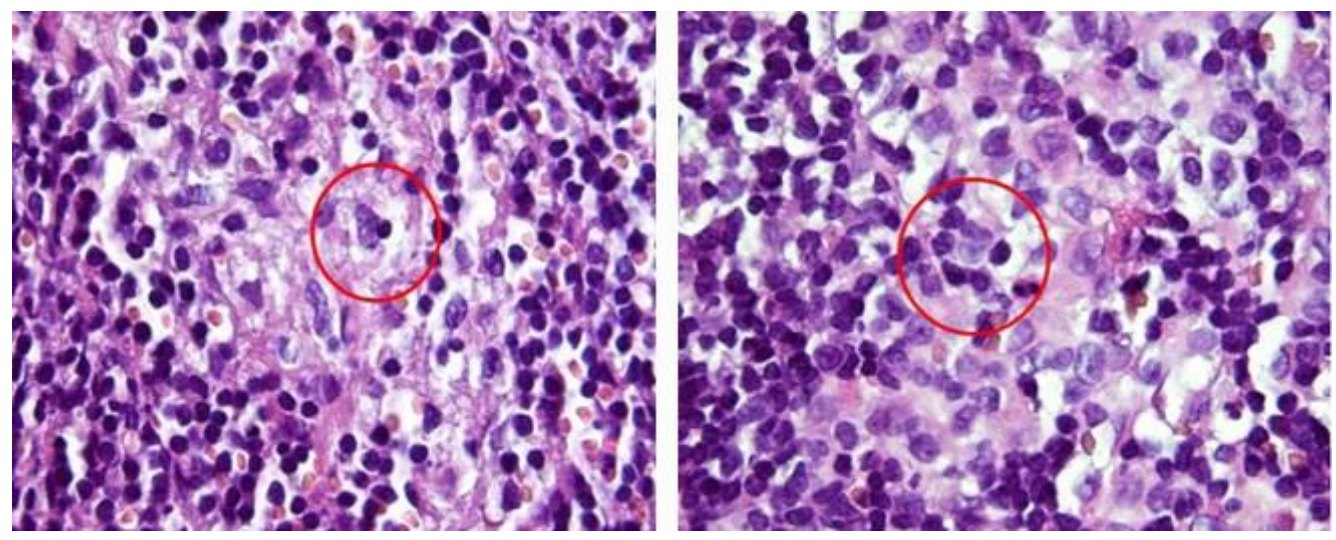

Figuras 3: Emperipolesis.

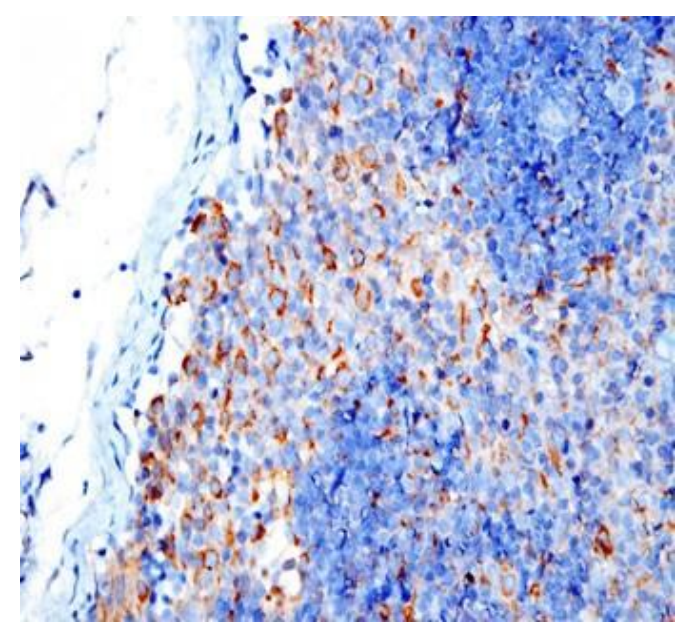

Figura 4: Inmunohistoquímica: Histiocitos marcados con CD68.

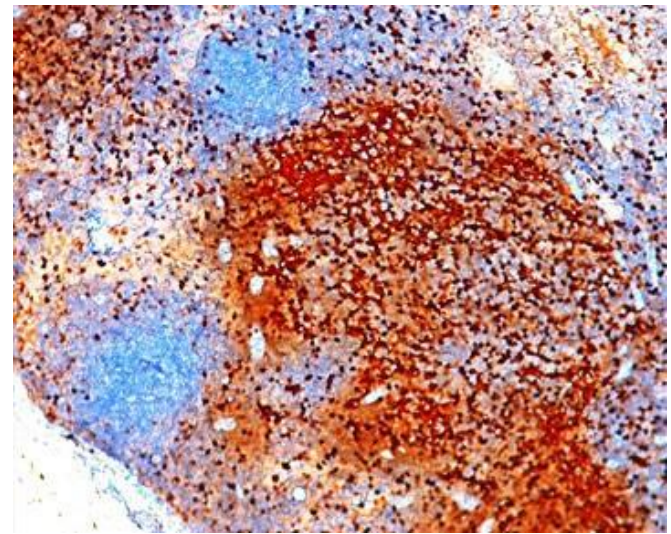

Figura 5: Inmunohistoquímica: Histiocitos marcados con S100 (coloración marrón).. 
se observaron además ocasionales macrófagos con emperipolesis (linfocitofagocitosis). No se observaron células atípicas en la muestra. Se realizó estudio de inmunohistoquímica que dio positivo para los marcadores S100 y CD68 mientras que CD1 fue negativo. Con estos hallazgos se llegó al diagnóstico de Histiocitosis sinusoidal o Enfermedad de Rosai Dorfman (Figuras 1 -5). .

\section{DISCUSIÓN}

La enfermedad de Rosai Dorfman o Histiocitosis Sinusal con Linfadenopatías Masivas (SHML por sus siglas en inglés) es una entidad clínica rara dentro de los trastornos de proliferación histiocítica. Se la clasifica dentro de las histiocitosis tipo II junto con enfermedades como como el xantogranuloma, reticulohistiocitoma y una forma familiar previamente designada como histiocitosis de Faisalabad (Morgan N. 2010). Con esta última existe una superposición fenotípica, sin embargo, se diferencian en que la histiocitosis de Faisalabad fue descrita como una nueva forma autosómica recesiva de histiocitosis en una familia altamente consanguínea originaria de Pakistán (Morgan N. 2010) quienes antes de desarrollar linfadenopatías e infiltración histiocítica padecían de deformidades articulares, sensoriales y pérdida auditiva. Las descripciones de SHML en la literatura muchas veces son casi siempre realizadas en base de casos aislados o en raras ocasiones series de pocos pacientes. Esta enfermedad fue descrita inicialmente en el año de 1969 por el grupo de Rosai y Dorfman (Rosai y Dorfman, 1969). Su epidemiología es poco clara con una incidencia global estimada en alrededor de 690 casos desde su descripción (https://rarediseases.org/rare-diseases/ rosai-dorfman-disease/). De acuerdo con la gran mayoría de observaciones la enfermedad suele presentarse en poblaciones pediátricas y en adultos jóvenes menores de 30 años con una predilección hacia los varones en un 58\% (Cai Yanan et al. 2017) (Piris, 2017), con una predilección por la etnia africana (Gomes Pinto et al. 2008), aunque existen excepciones anecdóticas donde la enfermedad es diagnosticada en edades más tardías, reportándose casos aislados entre la sexta y séptima década de vida (Liao et al. 2010). La enfermedad usualmente tiene una localización preponderantemente ganglionar, describiéndose compromiso extranodal en por lo menos algún órgano en $43 \%$ de los casos, mientras que solamente el $23 \%$ de los pacientes presentan exclusivamente afecciones extranodales (Cai Yanan et al. 2017). Como localizaciones extranodales más comunes se tiene a la piel y tejidos blandos (Mantilla
2016) con afección de forma muy inusual a nivel del tracto respiratorio superior e inferior, el tracto genitourinario, los ojos, las órbitas, los riñones, la tiroides, las mamas, los huesos y el páncreas donde a nivel de este órgano solamente se han reportado cuatro casos de compromiso en la literatura de habla inglesa (Mantilla 2016). Los pacientes con enfermedad extranodal tienen peor pronóstico que aquellos con enfermedad nodal (Mantilla 2016). Estas variaciones hacen del diagnóstico de esta enfermedad una tarea difícil.

La fisiopatología de la enfermedad no se encuentra claramente explicada puesto que no se ha identificado aún el gatillo para la proliferación histiocítica (https://rarediseases.org/rare-diseases/ rosai-dorfman-disease/). Se conoce que las células infiltrantes responsable de la enfermedad portan como marcadores celulares CD11c, CD14, CD33 y $\mathrm{S} 100$, mientras que los monocitos infiltrantes al parénquima ganglionar expresan también CD11b, CD16, CD36, lysozyme, Mac-387, y HLA-DR pero no S-100 (Paulli, 1992). El CD11c, antes considerado como un marcador exclusive de las células dendríticas actualmente ha sido identificado en ciertos macrofagos, especialmente en aquellos que provienen de ambientes inflamatorios (Cai Yanan et al. 2017). Observaciones adicionales han documentado también la presencia de proteínas relacionadas a células mieloides (MRP) 8 y MRP14, marcadores que pertenecen a la familia S100, en la superficie de las células de SHML, estos marcadores también son expresadas en grandes cantidades sobre la superficie de monocitos reclutados (Lagasse 1992). Esto sugiere que el origen de la infiltración histiocítica del Rosai Dorfman proviene de monocitos circulantes (Cai Yanan et al. 2017). Adicionalmente se han realizado observaciones donde se evidencia que la sobreexpresión de MRP8 y MRP14 detiene la diferenciación celular en células de estirpe monocítica, por lo que su presencia explicaría el porqué de la infiltración y proliferación exagerada de estas células en los tejidos comprometidos. Parecería ser que el desorden se asocia a un tipo fenómeno genético cuasi-neoplásico puesto que mutaciones mutuamente exclusivas del KRAS y MAP2K1 se han descrito en un tercio de los casos de la enfermedad (Piris 2017), lo que sugiere una proliferación relacionada a estos oncogenes.

Acerca de los posibles culpables para estas aberraciones celulares existen varias proposiciones. Algunos autores sugieren que podrían ser ciertos grupos de citoquinas proinflamatorias como lo son 
el factor estimulante de colonias de macrófagos, Il1, Il-6 y TNF- a (Cai Yan et al. 2017). Por el contrario, existen ciertos investigadores que propugnan un papel preponderante del virus herpético 6, el poliomavirus, Epstein-Barr, parvovirus B19, citomegalovirus y varicela zóster dentro del desarrollo de la enfermedad (Al-Daraji 2010) (Luppi 1998), mientras que otras líneas de investigación relacionan a la enfermedad con síndromes proliferativos de inmunoglobulinas, como es el caso del síndrome de IgG4 (Liu M, Li X, Li Y (2017). De forma creciente se ha encontrado una asociación entre la presencia de Rosai Dorfman y estados de compromiso inmunológico como los son las enfermedades linfoproliferativas, HIV, lupus eritematoso sistémico y amiloidosis AA generalizada (Cai Yan et al. 2017).

Se postula como explicación a este fenómeno que la disfunción inmunológica probablemente actúa de forma independiente o sinérgica para iniciar perturbación homeostásica, que conlleva a la formación de lesiones inflamatorias lo que a su vez actúa como gatillo para el reclutamiento de monocitos y por ende la patogénesis del Rosai Dorfman. (Rosai y Dorfman, 1972). Cuando analizó la literatura acerca de otros casos semejantes al expuesto, se utilizó PubMed realizando una búsqueda mesh con lo términos "Rosai Dorfman" AND "Toxoplasmosis" de la cual solamente se arrojaroncinco resultados relacionados, deloscuales solamente dos estudios correspondieron a reportes de caso semejantes al nuestro. La relación de esta enfermedad con la Toxoplasmosis se ha propuesto bajo la hipótesis de que una infección crónica oculta que podría provocar proliferación histiocítica debido a una exagerada respuesta inmune contra el antígeno que también podría verse favorecida por un fenómeno genético cuasi-neoplásico puesto que se han mutaciones mutuamente exclusivas del KRAS y MAP2K. (Liao et al. 2010)

La enfermedad tiene un comienzo insidioso y persistente con reagudizaciones clínicas frecuentes durante el curso de la entidad (Hazarika et al. 2000). Las manifestaciones clínicas son algo variables. sin embargo, se describe en la literatura de forma general la presencia de adenomegalias, las cuales se localizan habitualmente en las regiones cervicales, inguinales y torácicas asociadas a manifestaciones sistémicas inespecíficas como lo son astenia, poli mialgias y alza térmica. Adicionalmente la enfermedad puede cursar con una erupción cutánea de tipo papular de aparición intermitente, la cual se presenta en periodos de exacerbación clínica.
Al contrario de otras formas de histiocitosis el compromiso óseo es sumamente infrecuente (Samir et al. 2014) La enfermedad también se ha descrito como un proceso infiltrativo a nivel extraganglionar a nivel de tejidos tan variados como lo son la piel, pulmones y sistema nervioso central. Estas manifestaciones pueden coexistir de forma independiente con la enfermedad ganglionar y de acuerdo a ciertas observaciones parecerían no guardar relación entre sí. (Carpenter et al. 1978).

El diagnóstico de esta enfermedad se realiza mediante el estudio histopatológico del ganglio linfático, preferible mediante una biopsia excisional para observar toda su estructura. El hallazgo histológico más prominente es la expansión y dilatación de los sinusoides del ganglio linfático que están ocupados por abundantes histiocitos, estos tienen citoplasma vacuolado, con núcleos redondos de cromatina dispersa y ocasionales nucleolos. (Ashton-Key et al. 2018) Algunos histiocitos muestran en su citoplasma a linfocitos fagocitados (no degradados), este fenomeno de linfocitofagocitosis se conoce como emperipolesis, característico de esta enfermedad. Los cordones medulares del ganglio muestran abundantes células plasmáticas. Se observan centros germinales reactivos residuales, el parénquima ganglionar va perdiendo su estructura normal mientras más avanza la enfermedad. En las lesiones extranodales los histiocitos forman cúmulos simulando los sinusoides dilatados. En cuanto al inmunofenotipo, los histiocitos de Rosai Dorfman expresan S100 y marcadores asociados a macrofagos como CD4, CD11c, CD14, CD64, CD33, y CD68.

Los diagnósticos diferenciales en cuanto a la histopatología se hacen con la histiocitosis de células de Langerhans en donde los citoplasmas son menos claros, no se observa emperipolesis ni células plasmáticas. La histiocitosis reactiva y las neoplasias malignas sinusoidales, en estas dos no se observa emperipolesis, ni dilatación masiva de los sinusoides, además en las neoplasias se observan mitosis y atipia nuclear. (Arber et al. 2017). La enfermedad se considera como una entidad benigna sin embargo existen casos, especialmente aquellos con la presencia de grandes adenomegalias torácicas y abdominales que han requerido intervención quirúrgica con el fín de evitar cuadros compresivos. Dentro del diagnóstico diferencial de la enfermedad se debe sospechar de la presencia de desórdenes linfoproliferativos así como la presencia de algunos tipos de neoplasias como sarcomas de tejidos blandos en lo que respecta a su variante extraganglionar. 
Otras enfermedades a considerar incluyen las neoplasias histiociticas y el tumor tendosinovial de células gigantes. (Tataroglu et al. 2017)

\section{CONCLUSIONES}

La enfermedad de Rosai Dorfman es una entidad rara que cursa con adenomegalias sistémicas la cual puede ser confundida con otros diagnósticos altamente prevalentes en el medio ecuatoriano como tuberculosis ganglionar. Dado su comportamiento crónico con exacerbaciones agudas después de supuestos cuadros respiratorios virales de vías aéreas superiores asociadas a síntomas constitucionales, esta entidad debe ser considerada como un diagnóstico diferencial en pacientes con adenomegalias sistémicas de forma recurrente en nuestra población. La asociación de este síndrome con enfermedades crónicas como la toxoplasmosis se encuentra pobremente definida y se especula aun acerca de si existe una causalidad relacionada a dichos procesos.

\section{REFERENCIAS BIBLIOGRÁFICAS}

Al-Daraji W1, Anandan A, Klassen-Fischer M, Auerbach A, Marwaha JS, Fanburg-Smith JC. (2010) Soft tissue Rosai-Dorfman disease: 29 new lesions in 18 patients with detection of polyomavirus antigen in 3 abdominal cases. Ann Diagn Pathol 14: 309-31

Elaine Jaffe, Daniel A. Arber, Elias Campo Nancy, Lee Harris, Leticia Quintanilla-Fend. Hematopathology. Philadelphia, PA: Elsevier, [2017].

Ashton-Key, Margaret. Diagnostic Lymph Node Pathology Rosai-Dorfman disease. http://www .pathologyoutlines.com/ topic/breastmalignantrosaidorfman.html. PathologyOutlines.com website. Accessed May 18th, 2018.

Cai Yanan, Shi Zhangzhen, Bai Yuansong. Review of Rosai-Dorfman Disease: New Insights into the Pathogenesis of This Rare Disorder. Acta Haematol 2017;138: 14-23 DOI: 10.1159/000475588)

Carpenter RJ III, Banks PM, Mc Donald TJ, Sanderson DR. Sinus histiocytosis with massive Lymphadenopathy (Rosai-Dorfman disease): Report of a case with respiratory tract involvement. Laryngoscope 1978;88:1963-9

Gómez Pinto DC, Vidigal Tde A, Castro Bd, Santos
BH, Sousa NJ.. Rosai-Dorfman disease in the differential diagnosis of cervical lymphadenopathy. Rev Bras Otorrinolaringol 2008;74(4): 632-5

Lagasse E, Weissman. IL: Mouse MRP8 and MRP14, two intracellular calcium-binding proteins associated with the development of the myeloid lineage. Blood 1992; 79: 1907-1915

Liao HJ, Chiang CW. Toxoplasma IgG expressed in a patient with Rosai-Dorfman disease. Kaohsiung J Med Sci. 2010 Jul;26(7):373-6. doi: 10.1016/ S1607-551X(10)70061-9.

Liu M, Li X, Li Y (2017) Rosai-Dorfman disease with features of IgG4related disease in the breast: cases report and literature review. Asian Pac J Allergy Immunol 2018;36:51-57 DOI 10.12932/ AP0862.

Luppi M, Barozzi,P, Garber R (1998) Expression of human herpesvirus- 6 antigens in benign and malignant lymphoproliferative disease. Am J Pathol 153: 815-823.

Mantilla J, Goldeberg-Stein S, Wang Yanhua. 2016. Extranodal Rosai Dorfman Disease. Clinicopathologic Series of 10 patients with radiologic correlation and review of literatute. American Journal of Paathology; 145:211-22. doi:10.1093/ajcp/aqv029.

Morgan NV, Morris MR, Cangul H, Gleeson D, Straatman-Iwanowska A, Davies N, Keenan S, Pasha S, Rahman F, Gentle D, Vreeswijk MP, Devilee P, Knowles MA, Ceylaner S, Trembath RC, Dalence C, Kismet E, Köseoğlu V, Rossbach HC, Gissen P, Tannahill D, Maher ER.. 2010. Mutations in SLC29A3, Encoding an Equilibrative Nucleoside Transporter ENT3, Cause a Familial Histiocytosis Syndrome (Faisalabad Histiocytosis) and Familial Rosai-Dorfman Disease. PLoS Genet 6(2): e1000833. doi:10.1371/journal. pgen.1000833

National Organization for Rare Disorders. RosaiDorfman Disease. https://rarediseases.org/rarediseases/rosai-dorfman-disease/

Paulli M, Rosso R, Kindl S, Boveri E, Marocolo D, Chioda C, Agostini C, Magrini U, Facchetti F: Immunophenotypic characterization of the cell infiltrate in five case of sinus histiocytosis with massive lymphadenopathy (Rosai-Dorfman Disease). Hum Pathol 1992; 23: 647-654 
Piris MA, Aguirregoicoa E, Montes-Moreno S, Celeiro-Muñoz C. Castleman Disease and Rosai-Dorfman Disease. Seminars in Diagnostic Pathology (2017), https://doi.org/10.1053/j. semdp.2017.11.014

Samir Dalia, MD, Elizabeth Sagatys, MD, Lubomir Sokol, MD, PhD, and Timothy Kubal, MD. RosaiDorfman Disease: Tumor Biology, Clinical Features, Pathology, and Treatment. Cancer Control. October 2014, Vol. 21, No. 4

Rosai J, Dorfman RF. Sinus histiocytosis with massive lymphadenopathy: a newly recognized benign clinicopathologicalentity. Arch Pathol. 1969;87:63-70

Rosai J, Dorfman RF: Sinus histiocytosis with massive lymphadenopathy: a pseudolymphomatous benign disorder. Analysis of 34 cases. Cancer 1972; 30: $1174-1188$

Tataroglu et al. Soft Tissue Rosai-Dorfman Disease with Unusual Histopathologic Features: A Case Report J Clin Exp Pathol 2017, 7:6

Hazarika P, Nayak DR, Balakrishnan R, Kundaje HG, Rao PL. RosaiDorfman disease of the subglottis. J Laryngol Otol 2000;114:970-3

Gaitonde S. Multifocal, extranodal sinus histiocytosiswith massive lymphadenopathy: an overview. ArchPathol Lab Med 2007;131:1117-21 